\title{
Evaluation of stunned and infarcted canine myocardium by real time myocardial contrast echocardiography
}

P.M.M. Dourado, J.M. Tsutsui, W. Mathias Jr., J.L. Andrade, P.L. da Luz and A.C.P. Chagas
Instituto do Coração, Faculdade de Medicina,

Universidade de São Paulo, São Paulo, SP, Brasil

\section{Correspondence}

A.C.P. Chagas

Unidade de Aterosclerose

InCor, FM, USP

Av. Dr. Enéas Carvalho Aguiar, 44

05403-900 São Paulo, SP

Brasil

Fax: +55-11-3069-5447

E-mail: antonio.chagas@incor.usp.br

Research supported by Instituto do

Coração and Fundação Zerbini.

Publication supported by FAPESP.

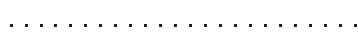

Received February 6, 2003 Accepted August 19, 2003

\begin{abstract}
Differentiation between stunned and infarcted myocardium in the setting of acute ischemia is challenging. Real time myocardial contrast echocardiography allows the simultaneous assessment of myocardial perfusion and function. In the present study we evaluated infarcted and stunned myocardium in an experimental model using real time myocardial contrast echocardiography. Sixteen dogs underwent 180 min of coronary occlusion followed by reperfusion (infarct model) and seven other dogs were submitted to 20 min of coronary occlusion followed by reperfusion (stunned model). Wall motion abnormality and perfusional myocardial defect areas were measured by planimetry. Risk and infarct areas were determined by tissue staining. In the infarct model, the wall motion abnormality area during coronary occlusion $\left(5.52 \pm 1.14 \mathrm{~cm}^{2}\right)$ was larger than the perfusional myocardial defect area $\left(3.71 \pm 1.45 \mathrm{~cm}^{2} ; \mathrm{P}<0.001\right)$. Reperfusion resulted in maintenance of wall motion abnormality $\left(5.45 \pm 1.41 \mathrm{~cm}^{2} ; \mathrm{P}=0.43\right.$ versus occlusion) and reduction of perfusional myocardial defect $\left(1.51 \pm 1.29 \mathrm{~cm}^{2}\right.$; $\mathrm{P}=0.004$ versus occlusion). Infarct size determined by contrast echocardiography correlated with tissue staining $(r=0.71 ; \mathrm{P}=0.002)$. In the stunned model, the wall motion abnormality area was $5.49 \pm$ $0.68 \mathrm{~cm}^{2}$ during occlusion and remained $5.1 \pm 0.63 \mathrm{~cm}^{2}$ after reperfusion $(\mathrm{P}=0.07)$. Perfusional defect area was $2.43 \pm 0.79 \mathrm{~cm}^{2}$ during occlusion and was reduced to $0.2 \pm 0.53 \mathrm{~cm}^{2}$ after reperfusion $(\mathrm{P}=$ 0.04). 2,3,5-Triphenyl tetrazolium chloride staining confirmed the absence of necrotic myocardium in all dogs in the stunned model. Real time myocardial contrast echocardiography is a noninvasive technique capable of distinguishing between stunned and infarcted myocardium after acute ischemia.
\end{abstract}

\section{Introduction}

After acute coronary occlusion, the restoration of epicardial blood flow results in a complex and variable myocardial reperfusion to the post-ischemic myocardium. Although flow to previously ischemic but vi-
Key words

- Myocardial perfusion

- Contrast echocardiography

- Myocardial stunning

- Myocardial infarction able tissue returns to normal, contractility remains altered due to myocardial stunning (1). The ability to determine whether regional left ventricular dysfunction is secondary to myocardial stunning or necrosis by a noninvasive method would be of important clinical value. 
Myocardial contrast echocardiography is an evolving method that has the potential to assess myocardial perfusion in patients with acute coronary artery disease (2). The method has been validated for the assessment of risk area and infarct size during total coronary occlusion in experimental models (3-6). However, previous techniques using intermittent harmonic imaging have demonstrated limitations regarding the wall motion analysis and time required for image acquisition $(2,5,7)$. Real time myocardial contrast echocardiography (RTMCE) is a recent technique that uses very low mechanical energy allowing the evaluation of microbubbles in the coronary circulation without their destruction, and simultaneous assessment of myocardial perfusion and function (7-10).

The present study was undertaken to determine by RTMCE the infarct size and stunned myocardium in an open-chest canine model of coronary occlusion and reperfusion, and their correlation with tissue staining.

\section{Methods}

\section{Animal preparation}

This study followed the guidelines of the "Position of the American Heart Association on Research Animal Use". Twenty-three mongrel dogs were anesthetized with sodium pentobarbital (30 mg/kg body weight, intravenously), intubated and mechanically ventilated. The heart was exposed through left lateral thoracotomy and suspended in a pericardial cradle. The proximal left anterior descending coronary artery was dissected free from surrounding tissue, a transit-time flow probe (Transsonic Systems, Inc., Ithaca, NY, USA) connected to a digital flowmeter was placed around the vessel and, at the time of coronary occlusion, a non-traumatic occluder was used. A $7 \mathrm{~F}$ catheter was positioned in the left carotid artery to monitor systemic arterial pressure (Biopac Systems, Goleta, CA, USA) and another catheter was introduced into the jugular vein for drug infusion.

\section{Study protocol}

Sixteen dogs weighing $17.5 \pm 3.6 \mathrm{~kg}$ were submitted to $180 \mathrm{~min}$ of left anterior descending coronary artery occlusion followed by $30 \mathrm{~min}$ of reperfusion (infarct model). Baseline RTMCE was obtained when hemodynamic stability had been achieved. The left anterior descending coronary artery was then occluded and a flowmeter signal was used to confirm complete coronary occlusion. At $180 \mathrm{~min}$ of occlusion, RTMCE was performed to determine the perfusional myocardial defect (PMD) and wall motion abnormality (WMA) areas by planimetry. RTMCE was repeated 30 min after reperfusion in order to assess infarct area and residual stunned myocardium. Hemodynamic data and epicardial left anterior descending coronary artery flow were determined at the same time as RTMCE acquisition.

In seven other dogs (mean weight $=20.5$ $\pm 4.5 \mathrm{~kg}$ ) the time of left anterior descending coronary artery occlusion was restricted to $20 \mathrm{~min}$ in order to cause ischemia without myocardial necrosis, followed by $30 \mathrm{~min}$ of reperfusion (stunned model). RTMCE, coronary flow measurements and hemodynamic data were obtained at baseline, at $20 \mathrm{~min}$ of coronary occlusion and at $30 \mathrm{~min}$ of reperfusion.

\section{Myocardial contrast echocardiography}

Echocardiographic images were acquired using a broadband 4-2-MHz transducer (HDI 5000, Philips Medical Systems Company, Bothell, WA, USA) in the longitudinal apical view. A water-filled latex interface was used to optimize imaging. Specific instrumentation settings of power pulse inversion imaging were: mechanical index ranging from 0.09 to 0.12 (usually 0.1 ), low dynamic range and maximal line density. After adjustment, 
these parameters were maintained constant for each experiment. Manually triggered, transient, high mechanical index imaging (flash) was deflagrated at peak contrast intensity to destroy microbubbles within the myocardium and allow subsequent myocardial replenishment $(7,11)$.

The contrast agent used was perfluorocarbon-exposed sonicated dextrose albumin (PESDA), that consisted of microbubbles containing decafluorobutane surrounded by an albumin shell, with a mean microbubble size of 3-5 $\mu \mathrm{m}$, and a concentration of $10^{9}$ microbubbles/ml (12). RTMCE was performed with continuous intravenous infusion of $0.1 \mathrm{ml} / \mathrm{kg}$ PESDA diluted in $80 \mathrm{ml}$ of normal saline at a rate of $2-4 \mathrm{ml} / \mathrm{min}$, injected through the jugular vein.

Left ventricular systolic function was visually assessed and WMA area was determined by planimetry using a software built into the echocardiographic system. After the flash, we evaluated the myocardial perfusion and a single contrast-enhanced end-diastolic frame was used for the measurement of PMD area at the time of maximal myocardial replenishment. The myocardial thickness of the interventricular septum and free wall segments was measured in the anatomical specimens and compared to two-dimensional echocardiography in order to obtain the best correlation between the anatomical slice and the echocardiographic frame.

Risk area was defined by RTMCE as the PMD area during left anterior descending artery occlusion, and in nine dogs it was compared to the myocardium at risk as determined by Evans blue staining. We considered infarct size determined by RTMCE to be the residual PMD area after coronary reperfusion, and compared it to the anatomical necrotic area determined by 2,3,5triphenyl tetrazolium chloride (TTC) staining (13). The stunned myocardium was defined as the area that remained with WMA but presented normal myocardial perfusion after reestablishment of coronary flow, with the absence of infarction confirmed by tissue staining. In the infarct model, the stunned area was calculated as WMA minus PMD area after 30 min of coronary reperfusion.

The images were stored on videotape and an optical disk for off-line processing and analysis. All measurements were performed by two blinded observers and interobserver variability about the data for all dogs of the infarct model was determined.

\section{Tissue staining}

At the end of each experiment, the heart was arrested with intravenous potassium chloride and excised. The left anterior descending coronary artery was again occluded at the same site and cannulated immediately after occlusion. The ostium of the right coronary artery and the main left branch were also cannulated. Evans blue dye $(1 \mathrm{mg} / \mathrm{kg})$ was infused through the catheter positioned in the right coronary artery and left main branch and a $2 \%$ solution of TTC was simultaneously injected into the left anterior descending coronary artery. Evans blue stained the regions not perfused by the left anterior descending coronary artery and was used to determine the risk area (unstained area). The heart was sectioned longitudinally and the slice corresponding to the echocardiographic view was incubated in TTC at $37^{\circ} \mathrm{C}$ for 30 $\mathrm{min}$. This technique stains viable myocardium brick red and does not stain necrotic tissue. Regions that failed to demonstrate brick red staining, appearing pale yellow, were considered to be infarcted myocardium (14-16). The stained slices were digitalized into an off-line computer. Risk areas, unstained by Evans blue, and necrotic, unstained by TTC, were measured by planimetry.

\section{Statistical analysis}

Continuous and normally distributed data are reported as mean \pm one standard devia- 
tion (SD) and qualitative data as proportions. Within-group comparisons were performed by the paired Student $t$-test, repeated-

Table 1. Hemodynamic data for the experimental dogs submitted to infarct or stunning.

\begin{tabular}{lccc}
\hline & HR $(\mathrm{bpm})$ & MAP $(\mathrm{mmHg})$ & LAD flow $(\mathrm{ml} / \mathrm{min})$ \\
\hline $\begin{array}{l}\text { Infarct model } \\
\text { Baseline }\end{array}$ & $135.4 \pm 19.5$ & $93.0 \pm 19.2$ & $21.6 \pm 6.8$ \\
$\quad$ LAD occlusion & $122.2 \pm 31.5$ & $91.7 \pm 23.1$ & $1.6 \pm 2.3^{+}$ \\
$\quad$ Reperfusion & $116.5 \pm 33.1^{*}$ & $88.6 \pm 29.3$ & $20.3 \pm 8.2^{*}$ \\
Stunned model & & & \\
$\quad$ Baseline & $135.0 \pm 26.5$ & $89.0 \pm 14.3$ & $22.1 \pm 7.5$ \\
LAD occlusion & $122.1 \pm 21.0$ & $84.3 \pm 12.8$ & $0.32 \pm 0.34^{+}$ \\
$\quad$ Reperfusion & $119.1 \pm 20.5^{*}$ & $80.6 \pm 13.3$ & $21.4 \pm 8.9^{*}$ \\
\hline
\end{tabular}

Data are reported as means $\pm S D$. $H R=$ heart rate; $L A D=$ left anterior descending coronary artery; MAP = mean arterial blood pressure.

${ }^{*} \mathrm{P}<0.05$ vs LAD occlusion; ${ }^{+} \mathrm{P} \leq 0.05$ vs baseline (Friedman test).

Figure 1. Wall motion abnormality (WMA) and perfusional myocardial defect (PMD) areas determined by real time myocardial contrast echocardiography at baseline, at $180 \mathrm{~min}$ of left anterior descending coronary artery (LAD) occlusion and after 30 min of reperfusion in the infarct model. After reperfusion there was maintenance of WMA area and reduction of PMD area. ${ }^{*} P<0.05$ for baseline $v s$ LAD occlusion; $+P<0.05$ for LAD occlusion vs reperfusion (ANOVA).

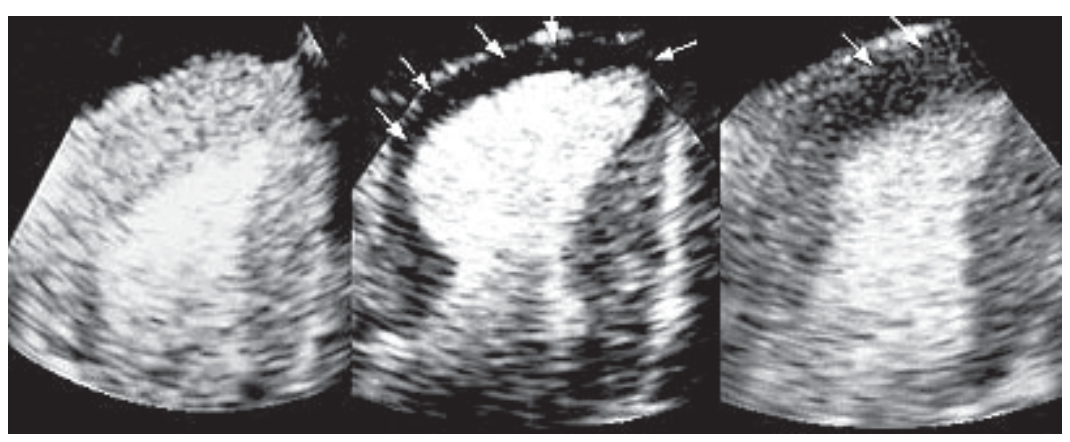

Figure 2. Representative example of real time myocardial contrast echocardiography in the infarct model showing normal myocardial perfusion at baseline (left) and the risk area during left anterior descending coronary artery occlusion (arrows) in the center. After 30 min of coronary reperfusion there was a clear reduction of perfusional defect size (arrows) and the infarcted area remained limited to the apical region (right). measures analysis of variance or Friedman test, as appropriate. Correlations between the RTMCE and anatomical data, as well as interobserver variability, were determined by linear regression using Spearman's rank statistic and agreement analysis. A P value $<0.05$ (two-sided) was considered to be statistically significant.

\section{Results}

Technically adequate data for wall motion and myocardial perfusion were obtained for all dogs. Hemodynamic data for the infarct and stunned models are shown in Table 1. Left anterior descending coronary artery flow was reduced to almost zero during occlusion. During the first minute of reperfusion, we observed a period of reactive hyperemia with an increase of 2.3 times (50.8 \pm $22.0 \mathrm{ml} / \mathrm{min}$ ) the baseline left anterior descending coronary artery flow in the infarct model, and of 2.6 times $(55.5 \pm 25.6 \mathrm{ml} / \mathrm{min})$ the baseline left anterior descending coronary artery flow in the stunned model. At the time of echocardiographic evaluation after reperfusion, left anterior descending coronary artery flow had returned to baseline levels.

\section{Infarct model evaluation}

At baseline, both left ventricular function and perfusion were normal in all dogs. We observed WMA soon after coronary occlusion that persisted until reperfusion. During coronary occlusion the WMA area (5.52 $\pm 1.14 \mathrm{~cm}^{2}$ ) was larger than the PMD area $\left(3.71 \pm 1.45 \mathrm{~cm}^{2} ; \mathrm{P}<0.001\right)$. After coronary reperfusion, there was maintenance of WMA area $\left(5.52 \pm 1.14 \mathrm{~cm}^{2}\right.$ during occlusion and $5.45 \pm 1.41 \mathrm{~cm}^{2}$ during reperfusion; $\mathrm{P}=$ 0.43 ) and a significant reduction of PMD area, from $3.71 \pm 1.45$ to $1.51 \pm 1.29 \mathrm{~cm}^{2}$; $\mathrm{P}=0.004$ (Figure 1).

During coronary occlusion, regions that failed to show contrast enhancement (risk 
area) were well defined and generally transmural. In contrast, after reperfusion regions that remained with PMD (infarct area) were irregular in shape and frequently subendocardial (Figure 2).

In nine dogs of the infarct model with Evans blue staining, RTMCE consistently underestimated the risk area, which was smaller than that determined by tissue staining $\left(4.58 \pm 1.30\right.$ vs $6.01 \pm 0.91 \mathrm{~cm}^{2} ; \mathrm{P}=$ $0.015)$. However, there was a good correlation between the RTMCE measurements and staining $(r=0.73 ; P=0.03$; Figure 3$)$.

Infarct size determined by RTMCE was $1.51 \pm 1.29 \mathrm{~cm}^{2}$ and necrotic area determined by TTC staining was $1.99 \pm 1.23 \mathrm{~cm}^{2}$ $(\mathrm{P}=0.29)$, with a strong linear correlation between these two measurements (necrotic area by TTC $=0.83 \mathrm{X}$ infarct size by RTMCE $\left.+0.73 \mathrm{~cm}^{2} ; \mathrm{r}=0.71 ; \mathrm{P}=0.002\right)$, as shown in Figure 4.

After $30 \mathrm{~min}$ of coronary reperfusion, myocardial perfusion was reestablished in the left anterior descending coronary artery territory, except in the infarcted areas. However, these regions continued to show impaired contractility even with normal perfusion. The stunned myocardium area (myocardium with WMA and without PMD) determined by RTMCE was $4.00 \pm 0.29 \mathrm{~cm}^{2}$. Tissue staining confirmed the absence of infarction in these areas.

\section{Stunned model evaluation}

In the stunned model the WMA area was $5.49 \pm 0.68 \mathrm{~cm}^{2}$ at $20 \mathrm{~min}$ of occlusion and $5.1 \pm 0.63 \mathrm{~cm}^{2}$ after reperfusion $(\mathrm{P}=0.07)$. PMD area was $2.43 \pm 0.79 \mathrm{~cm}^{2}$ during coronary occlusion and was reduced to almost zero $\left(0.2 \pm 0.53 \mathrm{~cm}^{2}\right)$ after reperfusion $(\mathrm{P}=$ 0.04) (Figure 5). TTC staining confirmed the absence of necrotic myocardium in all dogs of the stunned model.

Figure 6 demonstrates an example of normalization of perfusion with maintenance of WMA as observed in the stunned model.

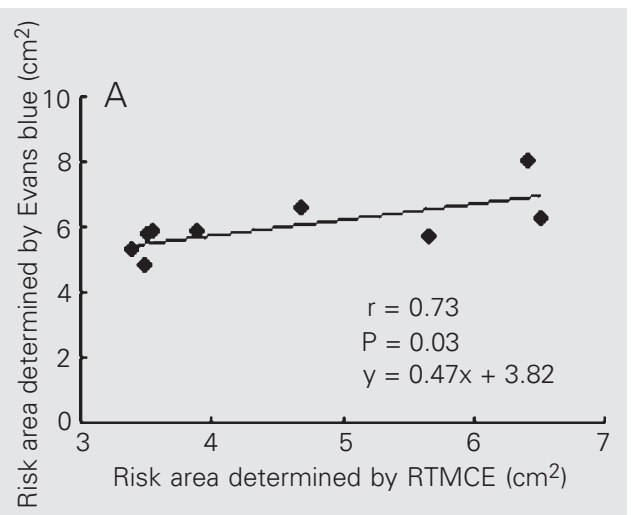

Figure 3. Correlation between risk areas determined by Evans blue staining and by real time myocardial contrast echocardiography (RTMCE) (A) and a Bland-Altman plot showing the mean difference (solid line) and limits of agreement (dashed lines) between measurements of risk area by the two methods (B).

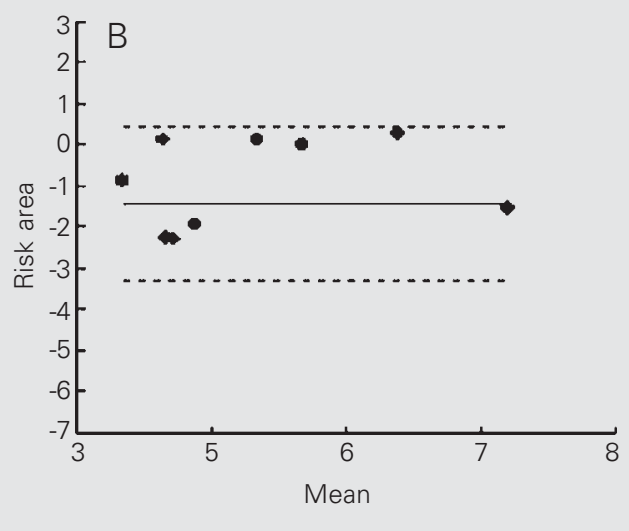

Figure 4. Correlation between infarct size determined by real time myocardial contrast echocardiography (RTMCE) and necrotic area determined by $2,3,5$ triphenyl tetrazolium chloride (TTC) staining (A) and a BlandAltman plot showing the mean difference (solid line) and limits of agreement (dashed lines) between infarct sizes determined by the two methods (B). 


\section{Interobserver variability}

We observed excellent agreement between the measurements of two observers for PMD area detected by RTMCE during coronary occlusion $(\mathrm{r}=0.91 ; \mathrm{P}<0.001)$, with a mean difference of -0.20 ( -1.35 to $0.95)$. Excellent agreement between two observers was also obtained after reperfusion $(r=0.96 ; P<0.001)$, with a mean difference of -0.03 (-0.40 to 0.34$)$.

\section{Discussion}

Although some studies have demonstrated the usefulness of low-energy RTMCE to

Figure 5. Wall motion abnormality (WMA) and perfusional myocardial defect (PMD) areas determined by real time myocardial contrast echocardiography at baseline, at 20 min of left anterior descending coronary artery (LAD) occlusion and after 30 min of reperfusion in the stunned model. After reperfusion there was maintenance of WMA area and reduction of PMD area to almost zero. ${ }^{*} \mathrm{P}<$ 0.05 for baseline vs LAD occlusion; $+\mathrm{P}<0.05$ for LAD occlusion $v s$ reperfusion (ANOVA).

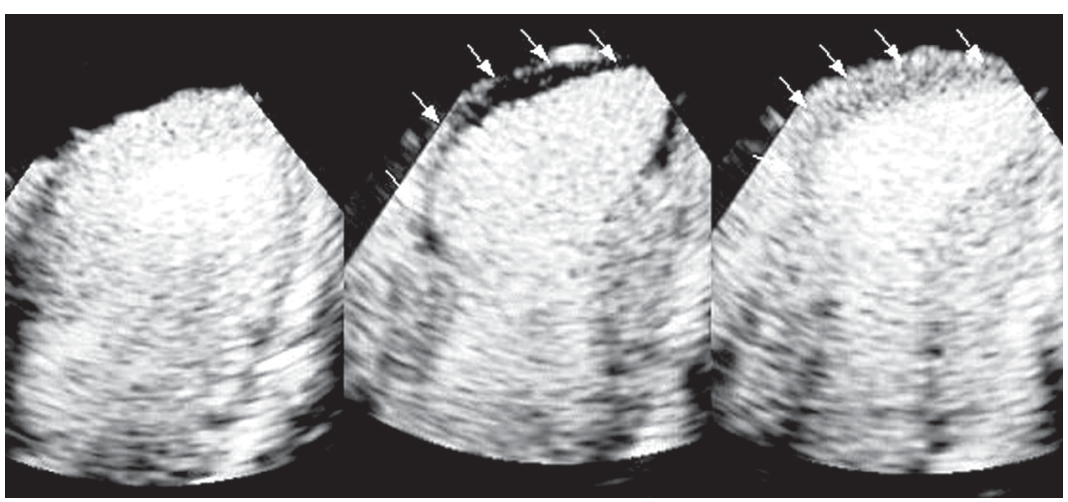

Figure 6. Representative example of real time myocardial contrast echocardiography in the stunned model showing normal myocardial perfusion at baseline (left) and risk area during left anterior descending coronary artery occlusion (arrows) in the center. After reperfusion (right) there was complete normalization of myocardial perfusion with maintenance of wall motion abnormality (arrows), demonstrating stunned myocardium. detect coronary stenosis and perfusional defects in the setting of acute myocardial ischemia $(9,10,17,18)$, its value for the simultaneous evaluation of myocardial function and perfusion in experimental models of infarction has not been yet described. The present study showed for the first time the progressive changes that occur with myocardial function and perfusion during coronary occlusion and after reperfusion, distinguishing normally perfused, collateral-dependent and non-perfused myocardium. We demonstrated that RTMCE accurately measured the areas of stunned and infarcted myocardium and underestimated the risk area when comparing to tissue staining.

The final infarct size is directly related to the risk area, which is also dependent on the duration of the ischemic period, collateral flow and efficacy of reperfusion $(19,20)$. Therefore, its measurement is an important parameter for clinical decisions in the setting of acute myocardial infarction. In the present study we observed that left anterior descending coronary artery occlusion resulted in a larger area of myocardial dysfunction than of perfusion defect in both models. A possible explanation for this result is the presence of collateral flow from adjacent vascular beds allowing some degree of myocardial perfusion in the margins of regions supplied by the occluded artery and decreasing the size of the risk area determined by RTMCE. This result is consistent with previous studies indicating that maturation of preexisting collateral vessels, as opposed to de novo growth of vessels, constitutes an early phase of collateral development in ischemic myocardium (21-23). Since we carried out the planimetry of perfusion defects by RTMCE only in the areas with total absence of myocardial contrast, regions with hypoperfusion surrounded the central ischemic area were not included in the PMD measurement. Although collateral-derived myocardial blood flow may not be adequate to maintain normal myocardial systolic func- 
tion distal to an occluded vessel, it may be sufficient to maintain viability for prolonged periods of coronary occlusion. Therefore, we should consider that the area with a perfusional defect during coronary occlusion as determined by RTMCE reflects the real area at risk for future necrosis.

In areas that are not supplied by collateral flow, prolonged coronary occlusion produces myocardial necrosis, leading to irreversible damage at the microvascular level $(24,25)$. If coronary reperfusion is achieved, either spontaneously or by means of thrombolysis or angioplasty, a variable amount of myocardium can be salvaged. Previous studies have demonstrated that infarct-related artery patency and myocardial perfusion are not necessarily concordant in humans $(26,27)$. Even with epicardial coronary reflow, myocardial perfusion may not be achieved (the lowreflow or no-reflow phenomenon) because of microvascular disruption, plugging by debris or myocardial edema $(14,28)$. Thus, the ability to identify the presence or absence of myocardial perfusion by myocardial contrast echocardiography during the post-acute myocardial infarct period may offer important advantages over simple assessment of infarct-related artery patency during catheterization, and predicts left ventricular function and further remodeling (29).

During the immediate period after acute infarction, the myocardium within the reperfused bed contains a mixture of both necrotic and stunned viable tissue (24). In the present study we showed that after reperfusion the contractility remained altered, with maintenance of the WMA area determined by RTMCE as compared to the occlusion period. On the other hand, there was a significant reduction of PMD area with complete normalization of perfusion in the stunned model and maintenance of the perfusion defect only in necrotic areas in the infarct model.

Therefore, RTMCE is a noninvasive method that allows simultaneous analysis of contractility and perfusion and was capable of demonstrating stunned (normal perfusion and maintenance of WMA) and infarcted tissue (absence of perfusion and depressed contractility). In the present study only three dogs presented discrete underestimation of infarct size by myocardial contrast echocardiography. One possible explanation for this result is that, because of the wave-front nature of myocardial damage following a prolonged period of ischemia, a mismatch between microvascular and myocyte necrosis can occur, resulting in areas of cellular death with relatively preserved perfusion. Irreversibly injured myocardium is generally associated with loss of microvascular integrity, which is proportional to the extension and severity of myocellular necrosis. In the poorly perfused areas, severe capillary damage and coagulation necrosis can be observed, but microvascular damage might also develop during reperfusion. Due to their ability to remain entirely within the intravascular space, microbubbles used as echocardiographic contrast agents are markers of blood flow and allow a noninvasive evaluation of microvascular integrity. However, when we analyzed all the dogs of the infarct model, there was no statistical difference between the infarct size predicted by RTMCE and that determined by TTC staining, showing that RTMCE can accurately measure the necrotic area.

\section{Limitations}

In the present study, risk area and infarct size were assessed by two-dimensional contrast echocardiography using a single tomographic plane, which was assumed to be representative of the whole left ventricle. We performed myocardial contrast echocardiography using only the apical longitudinal view in order to avoid posterior wall attenuation in the field farthest from the transducer that could occur when the short-axis plane is used. Consequently, the measurements ob- 
tained by RTMCE and anatomy represent an estimation of the true measurement of the spatial extent of risk area or infarcted myocardium. However, previous experimental studies have validated the good agreement between echocardiographic measurements and anatomic findings of risk area as well as infarct size using the methodology employed in this study $(20,30)$. The planimetry of WMA included areas with any degree of segmental wall motion, such as those presenting akinesia, hypokinesia or dyskinesia. We recognize that this approach does not consider qualitative changes in the segmental wall motion and could result in an underestimation of the improvement of wall motion after reperfusion. However, we noted that most segments presented akinesia soon after coronary occlusion that was maintained after reperfusion, without a significant change in the wall motion score index. In addition, we considered that a direct comparison between quantitative data of WMA and PMD was more suitable for the purpose of the study.

Another limitation is that visual assessment could lead to an overestimation of infarct size by RTMCE, which is based on the evaluation of myocardial replenishment after total destruction of microbubbles by a high emission power technique. Thus, borderline areas with some degree of residual perfusion could be interpreted as a contrast defect due to a late contrast replenishment.
In our study, infarct size was determined when there was full contrast replenishment of the normal areas but it is possible that borderline regions were included in the planimetry of contrast defect. Evaluation of myocardial blood flow by quantitative analysis could distinguish between areas with very low reflow and areas with no reflow.

\section{Clinical implications}

Main and co-workers (31) recently demonstrated in a small number of patients that full-motion myocardial contrast echocardiography and the power pulse inversion technique accurately predicted recovery of segmental function in patients with recent acute myocardial infarction. In the present study we demonstrate that RTMCE is a useful technique for visual assessment of stunned and infarcted myocardium. A rapid definition of the efficacy of reperfusion therapy as well as the extent of its residual microvascular damage has important implications for the treatment of acute myocardial infarction. It has been previously demonstrated that the presence of preserved microvascular flow in the post-reperfusion period is associated with a lower rate of fibrous scar and less ventricular remodeling (32-35). Thus the differentiation between stunned and infarcted myocardium by RTMCE has important clinical and prognostic implications.

\section{References}

1. Heyndrickx GR, Millard RW, McRitchie RJ, Maroko PR \& Vatner SF (1975). Regional myocardial functional and electrophysiological alterations after brief coronary artery occlusion in conscious dogs. Journal of Clinical Investigation, 56: 978-985.

2. Kaul S (1997). Myocardial contrast echocardiography: 15 years of research and development. Circulation, 96: 3745-3760.

3. Armstrong WF, Mueller TM, Kinney EL, Tickner EG, Dillon JC \& Feigenbaum H (1982). Assessment of myocardial perfusion abnormalities with contrast-enhanced two-dimensional echocardiography. Circulation, 66: 166-173.

4. Tei C, Sakamaki T, Shah PM, Meerbaum S, Shimoura S, Kondo S \& Corday E (1983). Myocardial contrast echocardiography: a reproducible technique of myocardial opacification for identifying regional perfusion deficits. Circulation, 67: 585-593

5. Villanueva FS, Glasheen WP, Skelenar J \& Kaul S (1993). Characterization of spatial patterns of flow within the reperfused myocardium by myocardial contrast echocardiography; implications for determining extent of myocardial salvage. Circulation, 88: 2596-2606.

6. White FC, Sanders M \& Bloor CM (1978). Regional distribution of myocardial blood flow after coronary occlusion and reperfusion in the conscious dog. American Journal of Cardiology, 42: 234-243.

7. Becker H \& Burns P (2000). Handbook of Contrast Echocardiography: Left Ventricular Function and Myocardial Perfusion. SpringerVerlag, New York.

8. Wei K, Jayaweera AR, Firoozan S, Linka A, Skyba DM \& Kaul S (1998). Quantification of myocardial blood flow with ultrasound- 
induced destruction of microbubbles administered as a constant venous infusion. Circulation, 97: 473-483.

9. Porter TR, Xie F, Silver M, Kricsfield D \& O'Leary E (2001). Real-time perfusion imaging with low mechanical index pulse inversion Doppler imaging. Journal of the American College of Cardiology, 37: 262-269.

10. Matsugata $H$, Peters B, Lafitte S, Strachan GM, Ohmori K \& DeMaria AN (2001). Quantitative assessment of myocardial perfusion during graded coronary stenosis by real-time myocardial contrast echocardiography. Journal of the American College of Cardiology, 37: 262269.

11. Pelberg RA, Wei K, Kamiyama N, Skelenar J, Bin J \& Kaul S (1999). Potential advantage of flash echocardiography for digital subtraction of b-mode images acquired during myocardial contrast echocardiography. Journal of the American Society of Echocardiography, 12: 85-93.

12. Porter TR \& Xie F (1995). Transient myocardial contrast following initial exposure to diagnostic ultrasound pressures with minute doses of intravenously injected microbubbles: demonstration and potential mechanisms. Circulation, 92: 2391-2395.

13. Jayaweera AR, Matthew TL, Skelenar J, Spotnitz WD, Watson DD \& Kaul S (1990). Method for quantitation of myocardial perfusion during myocardial contrast echocardiography. Journal of the American Society of Echocardiography, 3: 91-98.

14. Kloner R, Ganote CE \& Jennings RB (1974). The no-reflow phenomenon after temporary coronary occlusion in the dog. Journal of Clinical Investigation, 54: 1496-1508.

15. Fishbein MC, Meerbaum S, Rit J, Lado U, Kanmatsuse K, Mercier JC, Corday E \& Ganz W (1981). Early phase acute myocardial infarct size quantification: validation of the triphenyl tetrazolium chloride tissue enzyme staining technique. American Heart Journal, 101: 593-600.

16. Chagas ACP (1992). Proteção miocárdica em isquemia e reperfusão: estudo experimental sobre a preservação miocárdica e a ocorrência de fibrilação ventricular na reperfusão, em cães. Doctoral thesis, Faculdade de Medicina, Universidade de São Paulo, São Paulo, SP, Brazil.

17. Lafitte S, Matsugata H, Peters B, Togni M, Strachan M, Kwan OL \& DeMaria AN (2001). Comparative value of dobutamine and adenosine stress in the detection of coronary stenosis with myocardial contrast echocardiography. Circulation, 103: 2724-2730.

18. Lafitte $S$, Higashiyama A, Matsugata H, Peters B, Strachan M, Kwan OL \& DeMaria AN (2002). Contrast echocardiography can assess risk area and infarct size during coronary occlusion and reperfusion: experimental validation. Journal of the American College of Cardiology, 39: 1546-1554

19. Kemper AJ, O’Boyle JE, Cohen CA, Taylor A \& Parisi AF (1984). Hydrogen peroxide contrast echocardiography; quantification in vivo of myocardial risk area during coronary occlusion and of the necrotic area remaining after myocardial reperfusion. Circulation, 70: 309317.

20. Kaul S, Glasheen W, Ruddy TD, Pandian NG, Weyman AE \& Okada RD (1987). The importance of defining left ventricular area at risk in vivo during acute myocardial infarction: an experimental evaluation with myocardial contrast two-dimensional echocardiography. Circulation, 75: 1249-1260

21. Kaul S, Pandian NG, Guerrero JL, Gillam LD, Okada RD \& Weyman $A E$ (1987). Effects of selectively altering collateral driving pressure on regional perfusion and function in occluded coronary bed in the dog. Circulation Research, 61: 77-85.

22. Schaper J, Borges M \& Schaper W (1972). Ultrastructure of is- chaemia-induced changes in precapillary anastomotic network in the heart. American Journal of Cardiology, 29: 851-859.

23. Kaul S, Glasheen WP, Oliner JD, Kelly P \& Gascho JA (1991). Relation between antegrade blood flow through a coronary artery and the size of the perfusion bed it supplies: experimental and clinical implications. Journal of the American College of Cardiology, 17: 1403-1413

24. Reimer KA \& Jenning RB (1979). The wave front phenomenon of myocardial cell death. Laboratory Investigation, 40: 33-44.

25. Galiuto L, DeMaria AN, May-Newman K, Del Balzo U, Ohmori K, Bhargava V, Flaim SF \& lliceto S (1998). Evaluation of dynamic changes in microvascular flow during ischaemia-reperfusion by myocardial contrast echocardiography. Journal of the American College of Cardiology, 32: 1096-1101.

26. Ito $H$, Tomooka T, Sakai N, Yu H, Higashino $Y$, Fujii K, Masuyama T, Kitabatake A \& Minamino T (1992). Lack of myocardial perfusion immediately after successful thrombolysis: a predictor of poor recovery of left ventricular function in anterior myocardial infarction. Circulation, 85: 1699-1705.

27. Sabia PJ, Powers ER, Ragosta M, Sarembock IJ, Burwell LR \& Kaul $S$ (1992). An association between collateral blood flow and myocardial viability in patients with recent myocardial infarction. New England Journal of Medicine, 327: 1825-1831.

28. Johnson WB, Malone SA, Pantely GA, Anselone CG \& Bristow JD (1988). No reflow and extent of infarction during maximal vasodilation in porcine heart. Circulation, 78: 462-472.

29. Lincoff AM \& Topol EJ (1993). Illusion of reperfusion: does anyone achieve optimal reperfusion during acute myocardial infarction? Circulation, 88: 1361-1374.

30. Grayburn PA, Erickson JM, Escobar J, Womack L \& Velasco CE (1995). Peripheral intravenous myocardial contrast echocardiography using a $2 \%$ dodecafluoropentane emulsion: identification of myocardial risk area and infarct size in the canine model of ischaemia. Journal of the American College of Cardiology, 26: 13401347.

31. Main ML, Magalski A, Chee NK, Coen MM, Skolnick DG \& Good TH (2001). Full-motion pulse inversion power Doppler contrast echocardiography differentiates stunning from necrosis and predicts recovery of left ventricular function after acute myocardial infarction. Journal of the American College of Cardiology, 38: 1390-1394.

32. Agati L, Voci P, Bilotta F, Luongo R, Autore C, Penco M, lacoboni C, Fedele F \& Dagianti A (1994). Influence of residual perfusion within the infarct zone on the natural history of left ventricular dysfunction after acute myocardial infarction: a myocardial contrast echocardiographic study. Journal of the American College of Cardiology, 24: 336-342.

33. Agati L, Voci P, Luongo R, Testa G, Mallus MT, Di Roma A, Fedele F \& Dagianti A (1997). Combined use of dobutamine echocardiography and myocardial contrast echocardiography to predict recovery of regional dysfunction after coronary revascularization in patients with recent myocardial infarction. European Heart Journal, 18: 771 779 .

34. Ito H, Maruyama A, Iwakura K, Takiuchi S, Masuyama T, Hori M, Higashino Y, Fujii K \& Minamino T (1996). Clinical implications of the "no-reflow" phenomenon: a predictor of complications and left ventricular remodeling in reperfused anterior wall myocardial infarction. Circulation, 93: 223-228.

35. Agati L, Autore C, lacoboni C, Castaldo M, Veneroso G, Voci P, Fedele F \& Dagianti A (1999). The complex relation between myocardial viability and functional recovery in chronic left ventricular dysfunction. American Journal of Cardiology, 81: 33G-35G. 